Proceedings of the Edinburgh Mathematical Society (2002) 45, 411-420 (C)

DOI:10.1017/S001309150000122X Printed in the United Kingdom

\title{
SPECIAL PRECOVERS IN COTORSION THEORIES
}

\author{
KAREN D. AKINCI ${ }^{1}$ AND RAFAIL ALIZADE ${ }^{2}$ \\ ${ }^{1}$ Izmir University of Economics, Balcova Campus, Izmir, Turkey \\ (karen.akinci@izmirekonomi.edu.tr) \\ ${ }^{2}$ Department of Mathematics, Izmir Technology Institute, Gülbahçeköyü, \\ Urla, Izmir, Turkey (alizade@likya.iyte.edu.tr)
}

(Received 14 December 2000)

\begin{abstract}
A cotorsion theory is defined as a pair of classes Ext-orthogonal to each other. We give a hereditary condition (HC) which is satisfied by the (flat, cotorsion) cotorsion theory and give properties satisfied by arbitrary cotorsion theories with an HC. Given a cotorsion theory with an HC, we consider the class of all modules having a special precover with respect to the first class in the cotorsion theory and show that this class is closed under extensions. We then raise the question of whether this class is resolving or coresolving.
\end{abstract}

Keywords: cotorsion theory; hereditary condition; special precover; special pre-envelope

AMS 2000 Mathematics subject classification: Primary 18G15

Secondary 18E40

\section{Introduction}

Cotorsion theories were originally explored by Salce [7], who gave the following definition for cotorsion theories over $\mathbb{Z}$. The definition extends immediately to an arbitrary ring $R$.

Definition 1.1. A cotorsion theory is a pair $(\mathcal{F}, \mathcal{C})$ of classes such that

$$
\begin{aligned}
\mathcal{F} & ={ }^{\perp} \mathcal{C}=\left\{F \mid \operatorname{Ext}^{1}(F, C)=0 \text { for all } C \in \mathcal{C}\right\}, \\
\mathcal{C} & =\mathcal{F}^{\perp}=\left\{C \mid \operatorname{Ext}^{1}(F, C)=0 \text { for all } F \in \mathcal{F}\right\} .
\end{aligned}
$$

Hence the classes $(\mathcal{F}, \mathcal{C})$ satisfy the following conditions.

(1) $\operatorname{Ext}(F, C)=0$ for all $F \in \mathcal{F}$ and $C \in \mathcal{C}$.

(2) $\operatorname{Ext}(X, C)=0$ for all $C \in \mathcal{C}$ implies that $X \in \mathcal{F}$.

(3) $\operatorname{Ext}(F, X)=0$ for all $F \in \mathcal{F}$ implies that $X \in \mathcal{C}$.

$\mathcal{F}$ is called the cotorsion-free class and $\mathcal{C}$ is the cotorsion class.

By far the easiest and most common method of constructing a cotorsion theory is to generate or cogenerate it by some class $\mathcal{A}$ (or even set $\mathcal{A}$, see [5]). A cotorsion theory 
is said to be generated by a class $\mathcal{A}$ if it is of the form $\left({ }^{\perp} \mathcal{A},\left({ }^{\perp} \mathcal{A}\right)^{\perp}\right)$, that is the class ${ }^{\perp} \mathcal{A}$ is the cotorsion-free class generated by $\mathcal{A}$ and $\left({ }^{\perp} \mathcal{A}\right)^{\perp}$ is the cotorsion class generated by $\mathcal{A}$. Dually, a cotorsion theory is said to be cogenerated by the class $\mathcal{A}$ if it is of the form $\left({ }^{\perp}\left(\mathcal{A}^{\perp}\right), \mathcal{A}^{\perp}\right)$. The most studied cotorsion theory is the pair $(\mathcal{F}, \mathcal{C})$ of (torsion-free, cotorsion) groups found in $[\mathbf{2}, \mathbf{4}]$. For this example, it is not difficult to verify that the class $\mathcal{C}$ comes from the rationals $\mathbb{Q}$, where $\mathcal{C}$ and so $(\mathcal{F}, \mathcal{C})$ is seen to be cogenerated by the rationals. As an example of a cotorsion theory over an arbitrary ring we can extend the above example to the cotorsion theory $(\mathcal{F}, \mathcal{C})$, where $\mathcal{F}$ is the class of flat $R$-modules and $\mathcal{C}$ is the class of cotorsion $R$-modules (see [1] ).

Definition 1.2. For a left $R$-module $M$, a module $X$ in $\mathcal{X}$ is called an $\mathcal{X}$-envelope of $M$ if there is a linear map $f: M \rightarrow X$ such that the following hold.

(1) For any linear map $f^{\prime}: M \rightarrow X^{\prime}$ with $X^{\prime} \in \mathcal{X}$, there exists a linear map $g: X \rightarrow X^{\prime}$ such that $g \circ f=f^{\prime}$.

(2) If an endomorphism $g$ of $X$ is such that $g \circ f=f$, then $g$ is an automorphism.

In the case of (1) holding, but not necessarily (2), then $X$ is a pre-envelope.

Definition 1.3. For a left $R$-module $M$, a module $X$ in $\mathcal{X}$ is called an $\mathcal{X}$-cover of $M$ if there is a linear map $f: X \rightarrow M$ such that the following hold.

(1) For any linear map $f^{\prime}: X^{\prime} \rightarrow M$ with $X^{\prime} \in \mathcal{X}$, there exists a linear map $g: X^{\prime} \rightarrow X$ such that $f \circ g=f^{\prime}$.

(2) If an endomorphism $g$ of $X$ is such that $f \circ g=f$, then $g$ is an automorphism.

In the case of (1) holding, but not necessarily (2), then $X$ is a precover.

If $X$ is an $\mathcal{X}$-cover of $M$ and if $\mathcal{X}$ is closed under extensions, then $\operatorname{Ker} f \in \mathcal{X}^{\perp}$. Note that if $\operatorname{Ker} f \in \mathcal{X}^{\perp}$, then $X$ is an $\mathcal{X}$-precover (see [3] and [8, Lemma 2.1.1]).

Definition 1.4. An $R$-module $M$ has a special $\mathcal{X}$-pre-envelope $X$ if there is a monomorphism $f: M \rightarrow X$ with Coker $f \in{ }^{\perp} \mathcal{X}$.

Dually, the following definition can be given.

Definition 1.5. An $R$-module $M$ has a special $\mathcal{X}$-precover $X$ if there is an epimorphism $f: X \rightarrow M$ with Ker $f \in \mathcal{X}^{\perp}$.

The following definitions, given in [7] for abelian groups, are closely related to the above definitions.

Definition 1.6. A cotorsion theory $(\mathcal{F}, \mathcal{C})$ is said to have enough injectives if, for every module $M$, there exists a short exact sequence

$$
0 \rightarrow M \rightarrow C \rightarrow F \rightarrow 0
$$

where $C \in \mathcal{C}$ and $F \in \mathcal{F}$. 
Definition 1.7. A cotorsion theory is said to have enough projectives if, for every module $M$, there exists a short exact sequence

$$
0 \rightarrow C \rightarrow F \rightarrow M \rightarrow 0
$$

where $F \in \mathcal{F}$ and $C \in \mathcal{C}$.

Clearly $(\mathcal{F}, \mathcal{C})$ has enough injectives (projectives) if and only if every module has a special $\mathcal{C}$-pre-envelope (special $\mathcal{F}$-precover).

We shall refer to the above exact sequences as a special $\mathcal{C}$-pre-envelope and a special $\mathcal{F}$-precover of $M$, respectively.

The following proposition is proved in [7, Corollary 2.4] for abelian groups, but the proof can be easily extended to $R$-modules.

Proposition 1.8. A cotorsion theory has enough injectives if and only if it has enough projectives.

We will give a hereditary condition for a cotorsion theory $(\mathcal{F}, \mathcal{C})$ and prove under this condition that the class of all modules having special $\mathcal{F}$-precovers $(\mathcal{C}$-pre-envelopes $)$ is closed under extensions.

Definition 1.9. A class $\mathcal{A}$ of modules containing all projective (injective) modules is called resolving (coresolving) if, for every short exact sequence $0 \rightarrow A \rightarrow B \rightarrow C \rightarrow 0$, $B, C \in \mathcal{A} ;(A, B \in \mathcal{A})$ implies $A \in \mathcal{A}(C \in \mathcal{A})$.

Under some complementary conditions we prove that the above classes are resolving and coresolving.

\section{The hereditary condition}

For a general cotorsion theory over rings there are interesting results that we can obtain by generalizing results for the (flat, cotorsion) cotorsion theory. However there is a property that we must state first because the (flat, cotorsion) cotorsion theory satisfies a certain criterion, that of the following theorem, which may not be satisfied by a general cotorsion theory. We will call this the hereditary condition and denote it by HC.

Theorem 2.1. For any cotorsion theory $(\mathcal{F}, \mathcal{C})$ the following are equivalent.

(i) In any $0 \rightarrow A \rightarrow B \rightarrow C \rightarrow 0, A, B \in \mathcal{C}$ implies $C \in \mathcal{C}$.

(ii) In any $0 \rightarrow X \rightarrow Y \rightarrow Z \rightarrow 0, Y, Z \in \mathcal{F}$ implies $X \in \mathcal{F}$.

(iii) For any $C \in \mathcal{C}$ and $F \in \mathcal{F}, \operatorname{Ext}^{2}(F, C)=0$.

Proof. The proof is in four parts. We prove that each of (i) and (ii) is equivalent to (iii). 
(a) Assume (i), take any $C \in \mathcal{C}$ and $F \in \mathcal{F}$, and consider the short exact sequence formed from the injective envelope of $C: 0 \rightarrow C \rightarrow E \rightarrow K \rightarrow 0$. By (i), $K \in \mathcal{C}$. Now apply $\operatorname{Ext}(F,-)$ to it giving

$$
0=\operatorname{Ext}(F, K) \rightarrow \operatorname{Ext}^{2}(F, C) \rightarrow \operatorname{Ext}^{2}(F, E)=0 .
$$

Then $\operatorname{Ext}^{2}(F, C)=0$ for every $C \in \mathcal{C}$ and $F \in \mathcal{F}$.

(b) Assume $\operatorname{Ext}^{2}(F, C)=0$, for every $C \in \mathcal{C}$ and $F \in \mathcal{F}$, and take any short exact sequence $0 \rightarrow A \rightarrow B \rightarrow C \rightarrow 0$, where $A, B \in \mathcal{C}$, then apply $\operatorname{Ext}(F,-)$ to give

$$
0=\operatorname{Ext}(F, B) \rightarrow \operatorname{Ext}(F, C) \rightarrow \operatorname{Ext}^{2}(F, A)=0
$$

by assumption, so $\operatorname{Ext}(F, C)=0$ and we see that $C \in \mathcal{C}$.

(c) Assume (ii), take any $C \in \mathcal{C}$ and $F \in \mathcal{F}$, and consider the short exact sequence formed from the projective resolution of $F$ by cutting off at the first projective element and passing to the kernel of the epimorphism: $0 \rightarrow K \rightarrow P \rightarrow F \rightarrow 0$. By assumption (ii), $K \in \mathcal{F}$. Now apply $\operatorname{Ext}(-, C)$ to it giving

$$
0=\operatorname{Ext}(K, C) \rightarrow \operatorname{Ext}^{2}(F, C) \rightarrow \operatorname{Ext}^{2}(P, C)=0 .
$$

Then $\operatorname{Ext}^{2}(F, C)=0$ for every $C \in \mathcal{C}$ and $F \in \mathcal{F}$.

(d) Assume $\operatorname{Ext}^{2}(F, C)=0$, for every $C \in \mathcal{C}$ and $F \in \mathcal{F}$, and take any short exact sequence $0 \rightarrow X \rightarrow Y \rightarrow Z \rightarrow 0$, where $Y, Z \in \mathcal{F}$, then apply $\operatorname{Ext}(-, C)$ to give

$$
0=\operatorname{Ext}(Y, C) \rightarrow \operatorname{Ext}(X, C) \rightarrow \operatorname{Ext}^{2}(Z, C)=0
$$

by assumption, so $\operatorname{Ext}(X, C)=0$ and we see that $X \in \mathcal{F}$.

Definition 2.2. If a cotorsion theory satisfies the equivalent conditions of the above theorem, we will say that it satisfies the hereditary condition and denote this condition by HC.

As an aside it should be noted that the proposition, for any cotorsion theory $(\mathcal{F}, \mathcal{C})$ in $0 \rightarrow A \rightarrow B \rightarrow C \rightarrow 0$,

$$
(B, C \in \mathcal{C} \Rightarrow A \in \mathcal{C}) \quad \Leftrightarrow \quad(X, Y \in \mathcal{F} \Rightarrow Z \in \mathcal{F})
$$

is clearly not correct and this can be seen by the simple example below.

Example 2.3. Consider the cotorsion theory over groups $(\mathcal{G}, \mathcal{D})$ formed by the class of all abelian groups $G \in \mathcal{G}$ and all divisible groups $D \in \mathcal{D}$. Then obviously for all short exact sequences $0 \rightarrow A \rightarrow B \rightarrow C \rightarrow 0$, where $A, B \in \mathcal{G}$, we have that $C \in \mathcal{G}$. However, since every group has a divisible envelope, given a non-divisible group $H$ we can form its divisible resolution via its divisible envelope; $0 \rightarrow H \rightarrow D \rightarrow E \rightarrow 0$ with $D$ and $E$ divisible, forming a counterexample. 
Remark 2.4. One can easily prove that for any cotorsion theory $(\mathcal{F}, \mathcal{C})$ with the HC over a ring $R$, the following are equivalent.

(i) Every left $R$-module is in $\mathcal{C}$.

(ii) Every left $R$-module in $\mathcal{F}$ is also in $\mathcal{C}$.

(iii) Every left $R$-module in $\mathcal{F}$ is also in $\mathcal{P}$, the class of all projective modules.

\section{Special precovers and pre-envelopes}

The following theorem shows that the class of all modules having a special precover with respect to the first class in the cotorsion theory is closed under extensions. This is proved in [8, Theorem 3.1.8] for the (flat, cotorsion) cotorsion theory, when the ring $R$ is right coherent.

Theorem 3.1. Let $(\mathcal{F}, \mathcal{C})$ be a cotorsion theory with $H C$ and $0 \rightarrow A \rightarrow B \rightarrow C \rightarrow 0$ be exact. If $A$ and $C$ have special $\mathcal{F}$-precovers, then $B$ has a special $\mathcal{F}$-precover. Moreover, if $\mathcal{F}$ is closed under direct limits, then $B$ has an $\mathcal{F}$-cover.

Proof. Since $C$ has a special $\mathcal{F}$-precover $0 \rightarrow C_{1} \rightarrow F_{1} \rightarrow C \rightarrow 0$ with $C_{1} \in \mathcal{C}$ and $F_{1} \in \mathcal{F}$, we have the following pullback diagram:

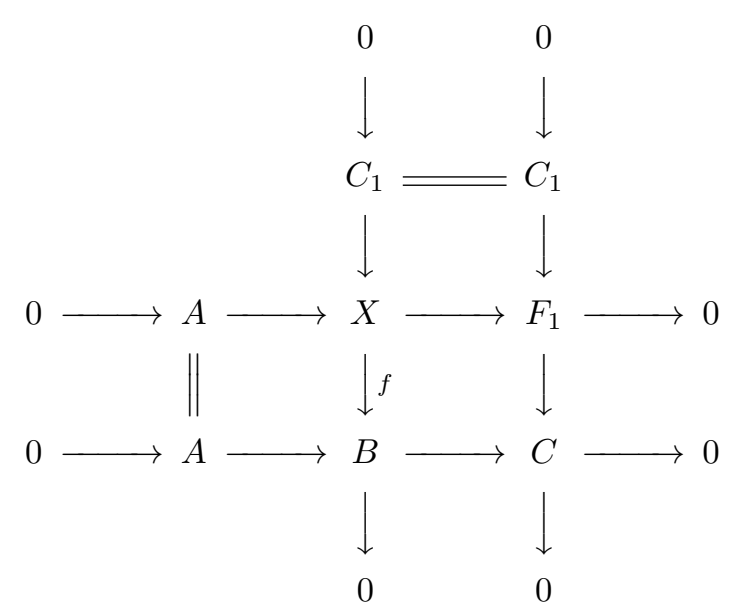

The module $A$ has a special $\mathcal{F}$-precover $0 \rightarrow C_{2} \rightarrow F_{2} \stackrel{g}{\rightarrow} A \rightarrow 0$ with $C_{2} \in \mathcal{C}$ and $F_{2} \in \mathcal{F}$. In the exact sequence

$$
\cdots \rightarrow \operatorname{Ext}\left(F_{1}, F_{2}\right) \stackrel{g_{*}}{\rightarrow} \operatorname{Ext}\left(F_{1}, A\right) \rightarrow \operatorname{Ext}^{2}\left(F_{1}, C_{2}\right) \rightarrow \cdots,
$$

we have $\operatorname{Ext}^{2}\left(F_{1} C_{2}\right)=0$ by assumption, and therefore $g_{*}$ is an epimorphism. So there is an exact sequence $E_{1}: 0 \rightarrow F_{2} \rightarrow Y \rightarrow F_{1} \rightarrow 0$, such that $g_{*}(E)=E_{1}$, that is we have 
the following commutative exact diagram:

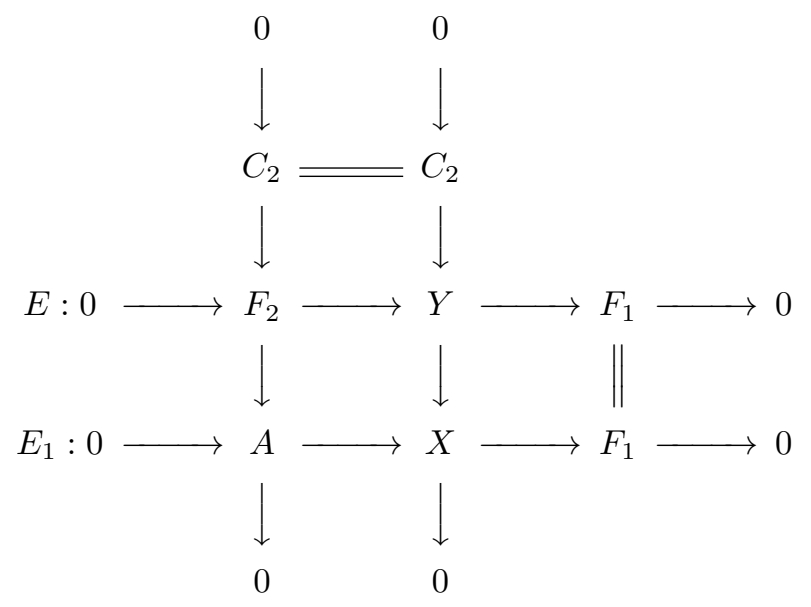

Since $F_{1}, F_{2} \in \mathcal{F}, Y \in \mathcal{F}$ also. From these two diagrams we have the following commutative exact diagram:

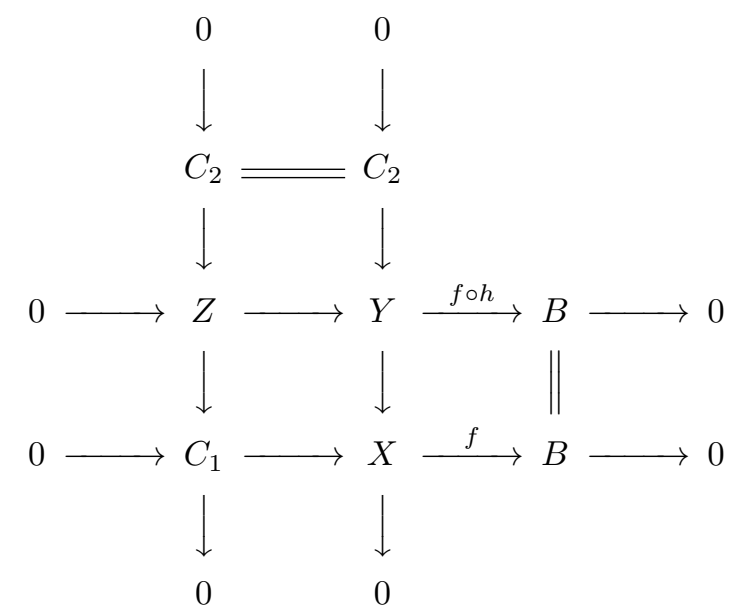

Now $Z \in \mathcal{C}$, since $C_{1}, C_{2} \in \mathcal{C}$. So $0 \rightarrow Z \rightarrow Y \rightarrow B \rightarrow 0$ is a special $\mathcal{F}$-precover for $B$. The second part of the theorem comes from $[\mathbf{3}]$.

Now we show that the classes of modules having special $\mathcal{F}$-precovers and special $\mathcal{A}$ pre-envelopes are resolving and coresolving.

Lemma 3.2. Suppose that every module from $\mathcal{F}$ has a special $\mathcal{C}$-pre-envelope and let $0 \rightarrow A \rightarrow F \rightarrow B \rightarrow 0$ be a short exact sequence with $F \in \mathcal{F}$, where $A$ has a special $\mathcal{C}$-pre-envelope. Then $B$ also has a special $\mathcal{C}$-pre-envelope. 
Proof. Let $0 \rightarrow A \rightarrow C \rightarrow D \rightarrow 0$ be a special $\mathcal{C}$-pre-envelope with $C \in \mathcal{C}$ and $D \in \mathcal{F}$. Then from the pushout diagram we have the following commutative and exact diagram:

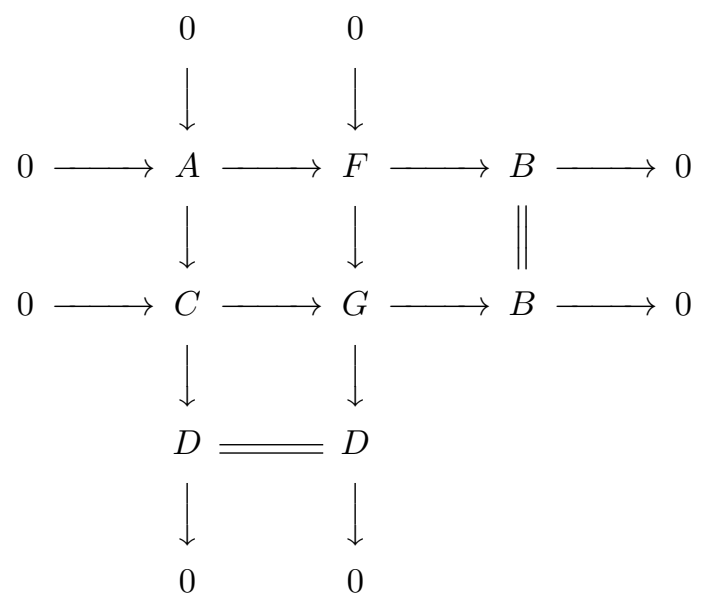

Now $G \in \mathcal{F}$, since $F, D \in \mathcal{F}$, and therefore $G$ has a special $\mathcal{C}$-pre-envelope $0 \rightarrow G \rightarrow$ $K \rightarrow M \rightarrow 0$ with $K \in \mathcal{C}, M \in \mathcal{F}$. We obtain the following commutative exact diagram:

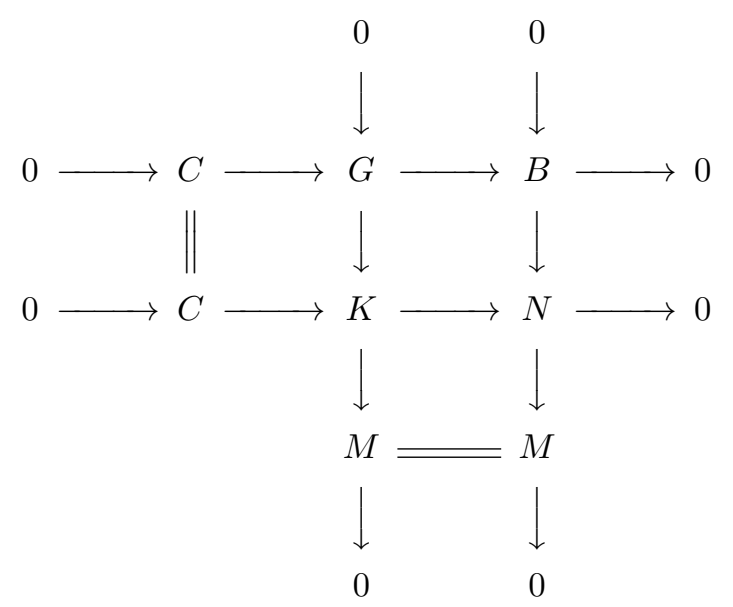

Then $N \in \mathcal{C}$, since $C, K \in \mathcal{C}$, and so the last column in the diagram gives a special $\mathcal{C}$-precover.

The dual statement can be proved in an analogous way.

Lemma 3.3. Suppose that every module from $\mathcal{C}$ has a special $\mathcal{F}$-precover and let $0 \rightarrow A \rightarrow C \rightarrow B \rightarrow 0$ be a short exact sequence with $C \in \mathcal{C}$, where $B$ has a special $\mathcal{F}$-precover. Then $A$ also has a special $\mathcal{F}$-precover.

Lemma 3.4. Let $0 \rightarrow A \rightarrow F \rightarrow B \rightarrow 0$ be a short exact sequence. If $F$ is flat and $A$ has a special $\mathcal{C}$-pre-envelope, then $B$ has a special $\mathcal{F}$-precover. 
Proof. Let $0 \rightarrow A \rightarrow C \rightarrow D \rightarrow 0$ be a special $\mathcal{C}$-precover with $C \in \mathcal{C}$ and $D \in \mathcal{F}$. Then from the pushout diagram we have the following commutative exact diagram:

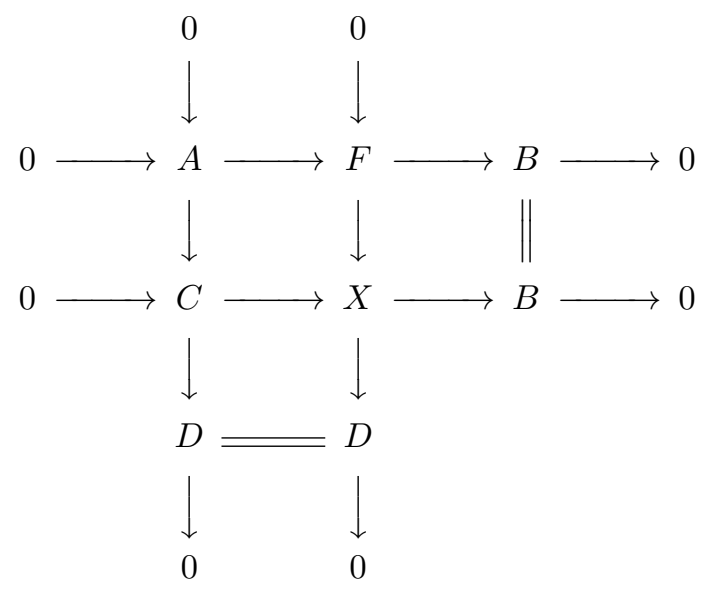

Since $F, D \in \mathcal{F}$, we have $X \in \mathcal{F}$, so $0 \rightarrow C \rightarrow X \rightarrow B \rightarrow 0$ gives a special $\mathcal{F}$-precover for $B$.

The dual statement can be easily formulated and proved.

Theorem 3.5. Suppose that the global dimension of $R, g l \cdot \operatorname{dim} R$, is finite. Then every module from $\mathcal{F}$ has a special $\mathcal{C}$-pre-envelope if and only if every module from $\mathcal{C}$ has a special $\mathcal{F}$-precover.

Proof. Suppose that every module from $\mathcal{F}$ has a special $\mathcal{C}$-pre-envelope and let $C$ be any module from $\mathcal{C}$. Since $g l \cdot \operatorname{dim} R<\infty, C$ has a finite projective resolution (see [6]):

$$
0 \rightarrow P_{n} \rightarrow P_{n-1} \rightarrow \cdots \rightarrow P_{0} \rightarrow C \rightarrow 0 .
$$

This sequence is the Yoneda product of the following short exact sequences:

$$
\begin{array}{ccccccccc}
0 & \rightarrow & P_{n} & \rightarrow & P_{n-1} & \rightarrow & K_{n-2} & \rightarrow & 0 \\
0 & \rightarrow & K_{n-2} & \rightarrow & P_{n-2} & \rightarrow & K_{n-3} & \rightarrow & 0 \\
& \vdots & & \vdots & & \vdots & & \vdots & \\
0 & \rightarrow & K_{1} & \rightarrow & P_{1} & \rightarrow & K_{0} & \rightarrow & 0 \\
0 & \rightarrow & K_{0} & \rightarrow & P_{0} & \rightarrow & C & \rightarrow & 0
\end{array}
$$

Now $P_{n}, P_{n-1}$ are projective and therefore they are in $\mathcal{F}$. By Lemma $3.2, K_{n-2}$ has a special $\mathcal{C}$-pre-envelope. Continuing in this way we see that $K_{0}$ has a special $\mathcal{C}$-preenvelope. Applying Lemma 3.3 to the last sequence we see that $C$ has a special $\mathcal{F}$ precover.

The proof of the inverse statement is dual.

Definition 3.6. If $g l \cdot \operatorname{dim} R<\infty$, the cotorsion theory $(\mathcal{F}, \mathcal{C})$ satisfies the $\mathrm{HC}$ and every module from $\mathcal{F}$ has a special $\mathcal{C}$-pre-envelope (or equivalently, every module from $\mathcal{C}$ has a special $\mathcal{F}$-precover), we will say that $(\mathcal{F}, \mathcal{C})$ satisfies the extended hereditary condition (denoted by EHC). 
Proposition 3.7. If the cotorsion theory $(\mathcal{F}, \mathcal{C})$ satisfies the $E H C$, then a module $M$ has a special $\mathcal{F}$-precover if and only if it has a special $\mathcal{C}$-pre-envelope.

Proof. Let $0 \rightarrow K \rightarrow F \rightarrow M \rightarrow 0$ be a special $\mathcal{F}$-precover of $M$ and $0 \rightarrow F \rightarrow C \rightarrow$ $L \rightarrow 0$ be a special $\mathcal{C}$-pre-envelope of $F$. Then we have the following commutative exact diagram:

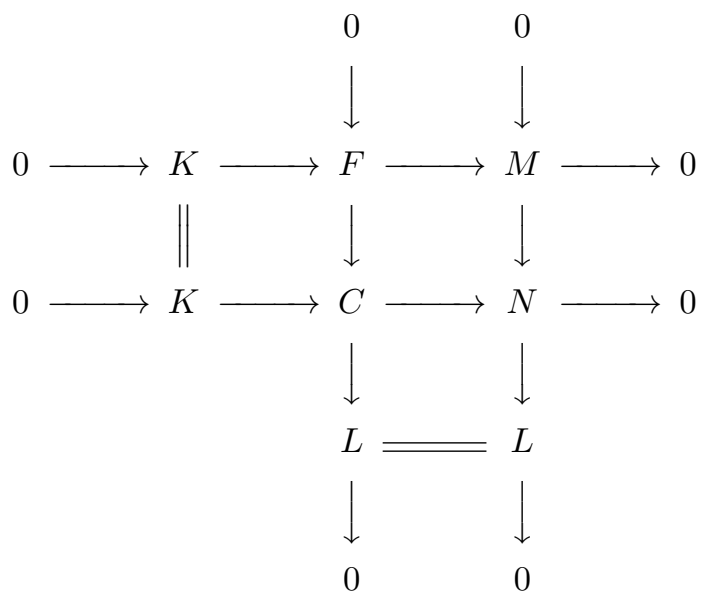

Now $N \in \mathcal{C}$, since $K, C \in \mathcal{C}$, and therefore the last column gives a $\mathcal{C}$-pre-envelope for $N$. The inverse statement can be proved in a dual manner.

Theorem 3.8. Suppose that the cotorsion theory $\mathcal{F}, \mathcal{C}$ satisfies the $E H C$ and $0 \rightarrow$ $A \rightarrow B \rightarrow C \rightarrow 0$ is a short exact sequence. If any two of the modules $A, B, C$ have a special $\mathcal{F}$-precover $(\mathcal{C}$-pre-envelope), then so does the third.

Proof. Suppose that $A, B$ have a special $\mathcal{F}$-precover. Then $A$ has a special $\mathcal{C}$-preenvelope by Proposition 3.7. Following the proof of Theorem 3.1.9 in [8], let $0 \rightarrow A \rightarrow$ $G \rightarrow D \rightarrow 0$ be a special $\mathcal{C}$-pre-envelope of $A$. Then we have the following commutative diagram:

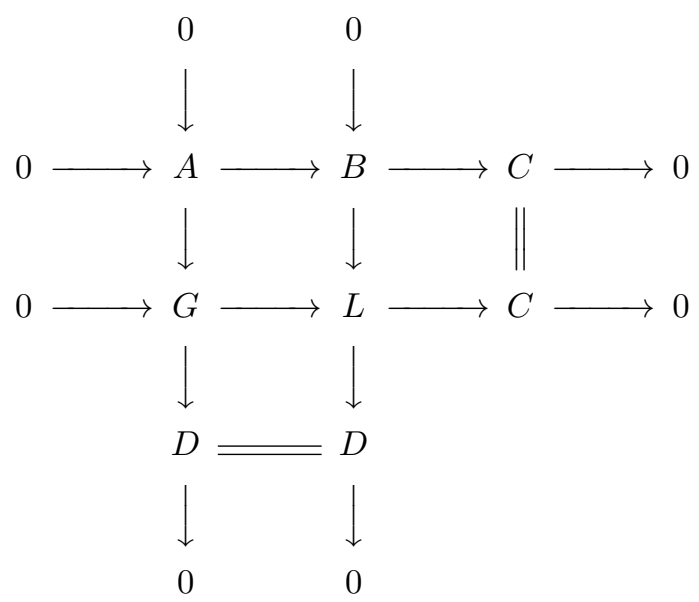


Since $B, D$ have special $\mathcal{F}$-precovers, by Theorem 3.1 the module $L$ also has a special $\mathcal{F}$-precover $0 \rightarrow K \rightarrow F \rightarrow L \rightarrow 0$. Then we have the following commutative diagram:

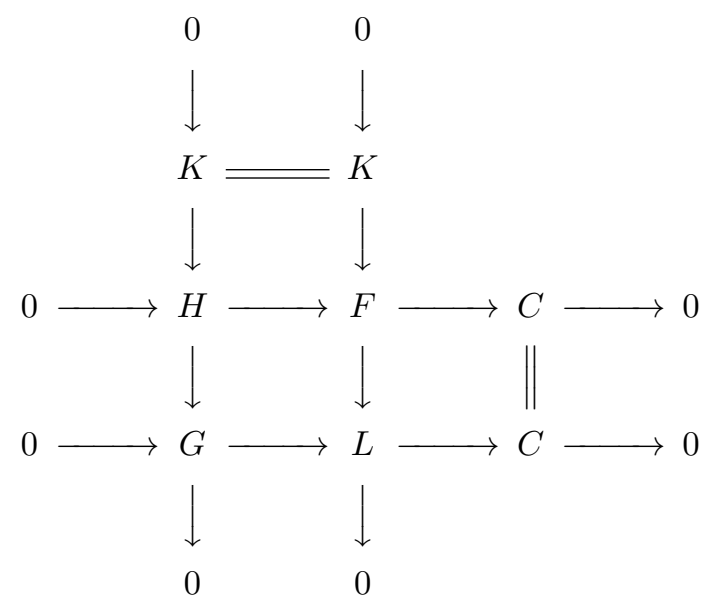

Since $K$ and $G$ are in $\mathcal{C}$, then $H$ is also in $\mathcal{C}$. So $0 \rightarrow H \rightarrow F \rightarrow C \rightarrow 0$ is a special $\mathcal{F}$-precover for $C$. Because of the previous proposition we can state that if $A, B$ have special $\mathcal{C}$-pre-envelopes, then $C$ also has a special $\mathcal{C}$-pre-envelope.

One can easily verify dually that if $B, C$ have a special $\mathcal{C}$-pre-envelope, then $A$ also has a special $\mathcal{C}$-pre-envelope. Now Proposition 3.7 and Theorem 3.1 complete the proof.

Acknowledgements. R.A. expresses gratitude for the support given to him by a grant from TUBITAK. A debt of thanks is also due to the referee for valuable comments and suggestions.

\section{References}

1. L. Bican, R. El Bashir And E. Enochs, All modules have flatcovers, Bull. Lond. Math. Soc., in press.

2. P. Eklof And J. Trilfaj, Making Ext vanish, Bull. Lond. Math. Soc. 33 (2001), 41-51.

3. E. ENochs, Injective and flat covers, envelopes and resolvents, Israel J. Math. 39 (1981), 189-209.

4. L. Fuchs, Infinite abelian groups (Academic, New York, 1970).

5. R. Gobel and S. Shelah, Cotorsion theories and splitters, Trans. Am. Math. Soc. 352 (2000), 5357-5379.

6. J. J. Rotman, An introduction to homological algebra (Academic, New York, 1979).

7. L. SAlCE, Cotorsion theories for abelian groups, Symp. Math. 23 (1972), 12-32.

8. J. Xu, Flat covers of modules, Lecture Notes in Mathematics, no. 1634 (Springer, 1996). 